\title{
Utilização do planejamento experimental na incorporação do resíduo da indústria cerâmica em argamassas para obtenção da capacidade de retenção de água
}

\section{(Use of the experimental design in the incorporation of the ceramic waste in mortars for obtaining water retention capacity)}

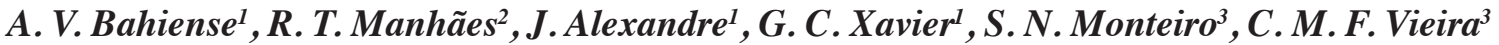 \\ ${ }^{1}$ Laboratório de Engenharia Civil, ${ }^{2}$ Laboratório de Física, ${ }^{3}$ Laboratório de Materiais Avançados \\ Universidade Estadual do Norte Fluminense Darcy Ribeiro \\ Av. Alberto Lamego, 2000, Campos dos Goytacazes, RJ 28013-600 \\ alexanvb@uenf.br, rosane.toledo@gmail.com
}

\begin{abstract}
Resumo
O município de Campos dos Goytacazes, RJ, possui um pólo cerâmico com mais de 100 indústrias cerâmicas e uma produção mensal estimada de 90 milhões de peças compreendidas entre lajotas, blocos e telhas. Muitos desses artefatos acabam gerando resíduos e impactos ambientais na região devido às falhas no processo produtivo e logístico das indústrias. Este trabalho propõe a incorporação do resíduo cerâmico em argamassas, avaliando as diversas proporções dos constituintes por meio do planejamento de experimentos em rede simplex buscando soluções técnicas, sem o tradicional empirismo existente na indústria, com o objetivo de alcançar um desempenho satisfatório da propriedade analisada, capacidade de retenção de água e, sem dúvida, favorecendo o desenvolvimento sustentável. O procedimento experimental foi baseado nas normas ABNT 13276, 13277 e 13281. A partir destes ensaios foram tomados dez pontos experimentais cujos teores máximos de cimento, areia e resíduo cerâmico são 20, 80 e 20\% em massa, respectivamente, que delimitou a superfície de resposta para as argamassas de índice de consistência $265 \pm 30 \mathrm{~mm}$. Este índice mostrou-se mais próximo da aplicação real para o modelo estatístico, porém, pouco acima do valor normalizado. O modelo em rede simplex cúbico especial mostrou melhor ajuste aos resultados experimentais, estimando respostas estatisticamente mais adequadas para as misturas. A superfície de resposta gerada apresentou capacidade de retenção de água da argamassa de 88 a 94,5\%, classificando-a como normal e alta, segundo a norma ABNT 13281. Mesmo com valor superior ao índice de consistência padrão (valor acima do estabelecido por norma) conseguiu-se uma capacidade de retenção padronizada com adição de resíduo na argamassa.
\end{abstract}

Palavras-chave: argamassa, resíduo cerâmico, planejamento experimental, rede simplex.

\begin{abstract}
The district of the Campos dos Goytacazes, RJ, Brazil, has a ceramic pole with more than 100 ceramic industries, with a 90 million monthly production (brick masonry, ceramic blocks and tiles). Due to failure in the productive industrial process, some of these materials have been generating wastes that produce environmental impacts. This work proposes the incorporation of the ceramic waste in mortars to evaluate the representative proportions through the lattice simplex experiments design, looking for technical solutions, without the industrial empiricism. These solutions could reach a satisfactory performance of the analyzed property, of the water retention capacity, and favor the sustainable development. The experimental procedure was based on standard test methods (ABNT 13276, 13277 e 13281). Starting from these lab tests, ten experimental points were taken with maximum content of cement, sand and ceramic waste 20, 80 and $20 \%$ mass, respectively, that delimited the surface response for the mortars with a consistence index around $265 \pm 30 \mathrm{~mm}$. This index means the real application for the statistical model, being however higher than the normalized value. The special cubic simplex model showed to best fit the experimental results leading to more statistically appropriate responses for the mixtures. The response surface generated showed a water retention capacity in mortar varying from 88 to $94.5 \%$, that could be classified as normal and high according to ABNT 13281. Even with the higher values than the standard consistence index (established value by the standard method), it was possible to obtain a standardized water retention capacity with the addition of ceramic waste into the mortar.
\end{abstract}

Keywords: mortar, ceramic waste, experimental design, simplex lattice.

\section{INTRODUÇÃO}

O município de Campos dos Goytacazes, RJ, tem como um dos pilares de sua economia a indústria de cerâmica vermelha. Este setor fornece, em sua grande maioria, produtos como blocos, lajotas e telhas. Como toda grande indústria, principalmente a de beneficiamento de matériasprimas naturais, gera um grande volume de resíduos sólidos. Estima-se que $10 \%$ do total produzido por estas cerâmicas é transformado em resíduo durante o processo de fabricação, transporte e estocagem, gerando aproximadamente $19 \mathrm{mil}$ toneladas/mês de resíduo [1]. 
A motivação principal deste trabalho é proporcionar um destino final economicamente viável e ambiental, de forma racional, aos resíduos provenientes do processo industrial de produção da indústria de cerâmica vermelha, que se encontram em grande disponibilidade na região Norte Fluminense. Portanto, utilizando ferramentas matemáticas para otimização de experimentos, pode-se determinar um número maior de proporções do resíduo na constituição das argamassas, permitindo obter melhores e mais amplas respostas experimentais (propriedade desejada), incluindo-se a modelagem numérico-experimental de misturas e análise estatística dos modelos matemáticos. Este trabalho visa buscar um modelo mais adequado de dosagem, considerando as misturas experimentais realizadas, principalmente com relação às misturas com resíduos, baseando-se na metodologia de modelagem numérico-experimental rede simplex (simplex-lattice design) [2,3].

\section{Planejamento de experimentos em rede simplex}

Alguns dos problemas encontrados ao realizar ensaios são decorrentes da necessidade de estudar simultaneamente o efeito dos fatores que constituem as argamassas, com diferentes níveis, o que torna os experimentos industriais inviáveis economicamente, visto que os custos e o tempo de execução são elevados [4]. As proporções dos materiais para realização dos ensaios foram calculadas matematicamente, e após o modelo numérico estabelecido para a resposta de interesse, seguiu-se uma comprovação experimental da eficiência dos modelos gerados por meio da análise de variância (ANOVA) [5].

\section{Modelagem numérica em rede simplex}

Em uma mistura, a quantidade qualquer total se mantém constante variando apenas seus componentes. Se a quantidade total é mantida constante, o valor da resposta varia quando mudanças são feitas nas proporções relativas dos componentes [2], conforme demonstrado pela equação A, onde $x_{i}$ representa a proporção do $i$-ésimo componente.

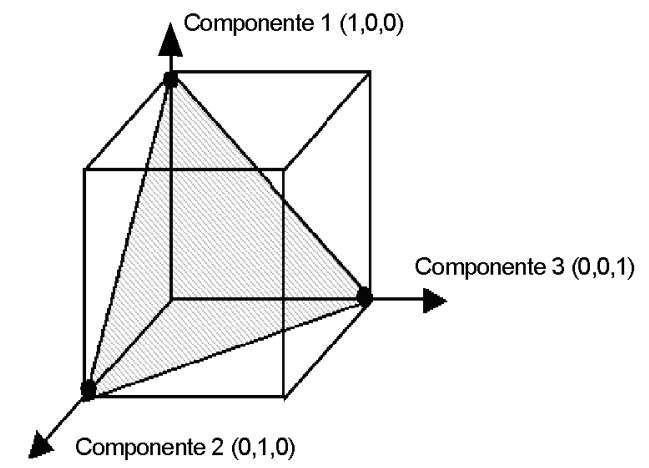

Figura 1: Rede simplex para três componentes (superfície de resposta) [2].

[Figure 1: Simplex lattice for three components (response surface) [2].]
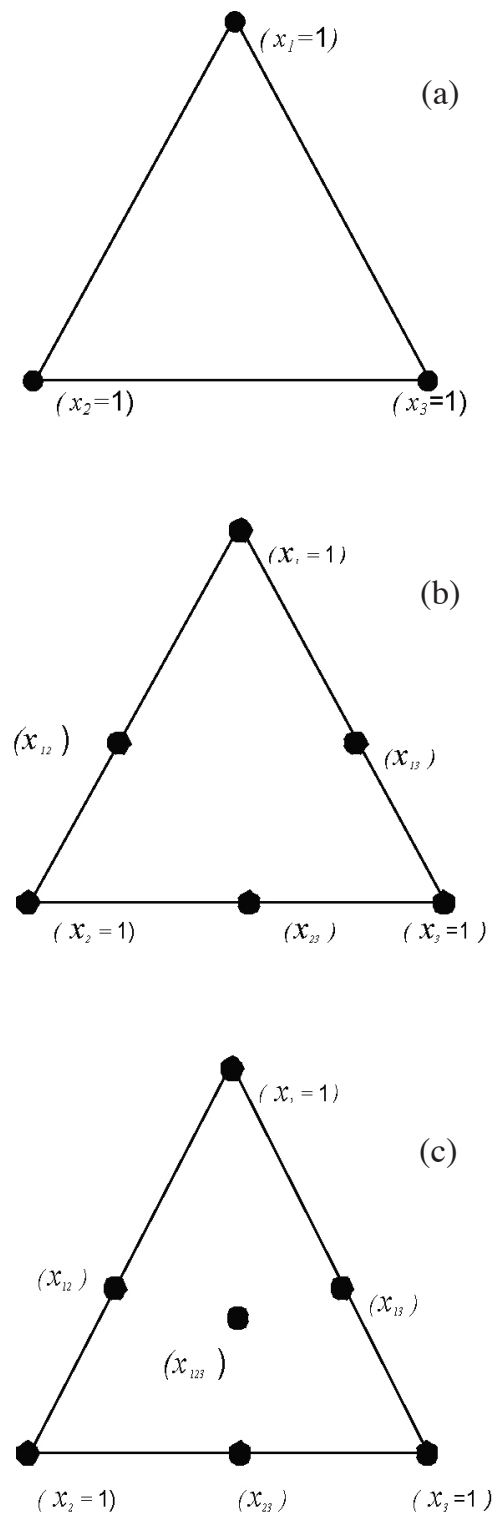

Figura 2: Pontos para a obtenção dos modelos. (a) linear, (b) quadrático, (c) cúbico especial.

[Figure 2: Points to obtaining models. (a) linear, (b) quadratic, (c) special cubic.]

$$
\sum_{i=1}^{\mathrm{q}} \mathrm{xi}=\mathrm{x}_{1}+\mathrm{x}_{2}+\ldots \ldots \ldots \ldots \ldots+\mathrm{x}_{\mathrm{q}}=1,0
$$

A Fig. 1 ilustra o fator espaço de três componentes $(q=3)$, e a equação A transforma-se em $x_{1}+x_{2}+x_{3}=1$.

A superfície de resposta e os pontos experimentais para os respectivos modelos matemáticos são representados pela Fig. 2.

\section{Representação matricial}

O sistema de misturas e respostas pode ser representado matricialmente na forma da equação B:

$$
\hat{y}=X b
$$


onde $\hat{y}$ e $b$ são as matrizes contendo os valores previstos pelo modelo para $y$ e as estimativas dos parâmetros, respectivamente. $X$ representa a matriz de composição das misturas. Sendo assim, os parâmetros $b$ são calculados resolvendo-se apenas uma equação matricial $\mathrm{C}$, podendo ser facilmente comprovado escrevendo as matrizes por extenso.

$$
X^{t} X b=X^{t} y
$$

Isolando-se a matriz $b$ têm-se, de forma direta, os valores da estimativa dos parâmetros. Se ampliar as matrizes $X$ e $y$ adequadamente, terá a solução geral para o ajuste de um modelo por mínimos quadrados, não importando quantos sejam as observações ou quantos parâmetros sejam necessários para caracterizar o modelo.

\section{Pseudocomponentes}

Há situações em que ocorrem restrições experimentais, como na formulação das argamassas, que devem conter simultaneamente os seus três componentes juntos, cimento, areia e resíduo. Isso é feito utilizando-se os pseudocomponentes originais que delimitam uma sub-região de interesse do simplex, onde o planejamento experimental é aplicado e a modelagem será válida [3].

Os pseudocomponentes $\left(x_{i}^{\prime}\right)$ definem as combinações dos componentes originais $\left(x_{i}\right)$ e delimitam uma subregião da superfície de resposta onde é possível realizar os experimentos de interesse. Desta forma, o somatório dos $\left(x_{i}\right)$ 's considerando as restrições experimentais não é igual à unidade [2], como mostra a equação D.

$$
\sum_{i=1}^{q} x i=x_{1}+x_{2}+\ldots+x_{q}<1,0
$$

Para mostrar como as restrições iniciais Lpseudocomponentes $\left(L_{i}\right)$ são definidos em termos dos componentes originais, temos a condição geral que o sistema consiste de $q$ componentes e $L_{i} \geq 0$ denota os limites inferiores para os componentes $i, i=1,2, \ldots, q$. Os limites inferiores são expressos na forma geral pela equação $E$ :

$$
0 \leq \mathrm{L}_{\mathrm{i}} \leq \mathrm{x}_{\mathrm{i}}, \quad \text { para } \mathrm{i}=1,2, \ldots, \mathrm{q}
$$

onde alguns dos $L_{i}$ poderiam ser nulos. Se subtrair dos limites inferiores $L_{i}$ de $x_{i}$ e dividir a diferença por $\left[1-\left(\Sigma L_{i}\right)\right]$, tem-se os L-pseudocomponentes $\left(x_{i}^{\prime}\right)^{\text {ss }}$ definidos usando-se a transformação da equação $\mathrm{F}$ :

$$
\begin{aligned}
& x_{i}^{\prime}=\frac{x_{i}-L_{i}}{1-L} \\
& \text { sendo, } L=\sum_{i=1}^{q} L i<1 .
\end{aligned}
$$

$\mathrm{Na}$ análise do modelo estatístico e para obtenção das matrizes de misturas, foi utilizado o software Statistica ${ }^{\circledR}$. Para a elaboração da superfície de resposta das misturas através da rede simplex, utilizando o modelo estatístico cúbico especial, com o software Surfer Version 5.0, Golden Software.

\section{MATERIAIS E MÉTODOS}

\section{Materiais}

As argamassas convencionais geralmente têm como seus constituintes básicos, cimento, areia e cal hidratada. A proposta deste trabalho é a incorporação do resíduo cerâmico em substituição total a cal e parcial a areia.

Utilizou-se areia de quartzo natural do rio Paraíba do Sul, por ser na prática a mais usualmente empregada no preparo de argamassas em Campos dos Goytacazes. O material foi caracterizado segundo as normas técnicas [6,7]. A areia passou previamente por um processo de secagem em estufa a $105^{\circ} \mathrm{C}$ por $24 \mathrm{~h}$. Após a secagem foram adotadas as frações granulométricas da areia normal para ensaio, segundo os procedimentos de separação da ABNT [8].

Foi utilizado como aglomerante o cimento portland compostos CPII E 32 Votaram, por ser o mais utilizado na região de Campos dos Goytacazes, lote $\mathrm{N}^{\circ}$. L-0092-K$06 / 215$.

O resíduo utilizado neste trabalho é proveniente de cerâmicas distintas de Campos dos Goytacazes, e foi recolhido obedecendo aos procedimentos de amostragem de resíduos sólidos [9]. Este resíduo foi coletado em estado seco ao ar em depósitos provisórios anexos às empresas.

\section{Métodos}

O resíduo cerâmico foi caracterizado segundo seu tamanho de partícula, composição química e mineralógica.

$\mathrm{O}$ resíduo coletado encontrava-se em formas e tamanhos variados e excediam os diâmetros máximos de agregados utilizados em argamassas. Desta forma, optou-se pela sua cominuição, o que foi feito por processo de moagem em um moinho de bolas Sonnex Contenco. Para se estabelecer o tempo ideal de moagem e obter uma boa resposta de finura, com baixo consumo energético, foram adotados períodos de $30 \mathrm{~min}, 1 \mathrm{~h}, 1,5 \mathrm{~h}$ e $2 \mathrm{~h}$. Os tempos foram arbitrados após análise visual dos fragmentos, buscando estipular um tempo ideal em função da eficiência do moinho e tamanho das partículas. O módulo de finura (MF) é utilizado para classificar o agregado miúdo (areia) e é definido como o somatório das porcentagens acumuladas retidas nas peneiras da série normal, dividido por 100. A classificação quanto ao módulo de finura é definida como: Areia fina $-\mathrm{MF}<2,4$, Areia média $-2,4<\mathrm{MF}<3,9$, Areia grossa $-\mathrm{MF}>3,9$.

A determinação da composição química semiquantitativa do resíduo cerâmico, em forma de óxidos, foi obtida por meio da técnica de análise química por fluorescência de raios $\mathrm{X}$, de energia dispersiva (EDX), em um equipamento Shimadzu EDX-700. A condição de ajuste foi a de "vácuo dois canais" e as amostras foram analisadas em forma de pó. O resíduo passou por secagem em estufa a $105^{\circ} \mathrm{C}$ por 
$24 \mathrm{~h}$. Após este período as amostras foram destorroadas em almofariz para redução de partículas maiores e, em seguida, moídas em moinho de bolas Pulzerisette 5 Fritsch, sendo em seguida peneirada na malha ABNT 200 (abertura 0,075 $\mathrm{mm})$.

As argilas (matérias-primas) foram analisadas por diversos pesquisadores $[5,10-12]$ em estudos que mostram que o argilomineral predominante em sua constituição é a caulinita. Essas matérias-primas da forma como são industrializadas para formação dos artefatos da indústria de cerâmica vermelha, passam por um processo térmico que altera a constituição de alguns de seus minerais, como no caso da caulinita que, após certas transformações térmicas que se iniciam a $490{ }^{\circ} \mathrm{C}$ [12] gera a metacaulinita, que é um aluminosilicato de estrutura desordenada, que apesar de não possuir, por si só, propriedades aglomerantes, reage quimicamente em presença de água com o hidróxido de cálcio $(\mathrm{CH})$, originando novos compostos hidratados com propriedades cimentícias e insolúveis em água [12]. A metacaulinita (material pozolânico), quando adicionada à argamassa entrará em contato com a portlandita liberada na hidratação do cimento portland e passa a ter propriedades cimentícias. Dessa forma, parte do resíduo cerâmico considerado filler pode comportar-se também como material aglomerante. O filler é um agregado fino, constituído de particulas minerais de dimensões inferiores a $0,075 \mathrm{~mm}$. Além de contribuir positivamente para as propriedades tecnológicas dos produtos à base de cimento portland, a pozolana (metacaulinita) servirá como alternativa também de economia em zonas dentro da matriz cimentícia com carência de cimento.

Atualmente existe uma tendência em conjugar os efeitos benéficos e específicos de diferentes aditivos minerais para incrementar as propriedades do concreto e argamassas e sua durabilidade, aglutinando-se a alta reatividade com a melhoria proporcionada na reologia do concreto fresco e argamassa [13], no caso deste estudo, a capacidade de retenção de água.

Foram utilizados três modelos numéricos para a determinação das superfícies de resposta, conforme a quantidade mínima de pontos para a geração dos mesmos: modelo linear ( 3 pontos), modelo quadrático ( 6 pontos) e modelo cúbico especial (7 pontos). As restrições experimentais adotadas, conforme a imposição do índice de consistência de $265 \pm 30 \mathrm{~mm}$, foram $x_{1} \geq 0,10 ; x_{2} \geq 0,7$ e $x_{3} \geq 0,10$, onde $x_{1}, x_{2}$ e $x_{3}$ representam respectivamente o componente cimento, areia e resíduo cerâmico. Os limites das proporções dados em massa dos componentes, em relação à unidade, são expressos por $0,10 \leq x_{1} \leq 0,20 ; 0,70 \leq$ $x_{2} \leq 0,80$ e $0,10 \leq x_{3} \leq 0,20$.

A resposta experimental avaliada neste trabalho é a propriedade de capacidade de retenção de água [14]. Para cada ponto experimental foram feitas três repetições por ensaio. Em virtude do tamanho de partícula do resíduo, que absorve grande quantidade de água, foi adotado o índice de consistência $265 \pm 30 \mathrm{~mm}$ para a determinação do teor de água a ser adicionado [15]. Este valor é superior ao índice de consistência padrão para argamassas com materiais convencionais $(255 \pm 10 \mathrm{~mm})$, pois não foi possível experimentalmente obter argamassa com o índice padrão adicionando resíduo cerâmico. Usou-se 10 pontos experimentais, tanto para a montagem dos modelos matemáticos quanto para os testes de ajuste e análise estatística. Em cada ponto do planejamento experimental realizaram-se três repetições, a fim de obter maior representatividade dos resultados.

\section{RESULTADOS E DISCUSSÃO}

A areia utilizada neste trabalho possui módulo de finura 2,65, classificando-a como areia média, massa específica aparente no estado solto $1,39 \mathrm{~g} / \mathrm{cm}^{3}$ (considera os vazios entre partículas) e massa específica teórica de $2,65 \mathrm{~g} / \mathrm{cm}^{3}$ [16]. O cimento possui uma área especifica maior que $260 \mathrm{~m}^{2} / \mathrm{kg}$ [17].

O resíduo cerâmico foi moído para a fixação do tamanho de partícula na mistura. A Fig. 3 mostra as curvas de distribuição do tamanho de partículas correspondentes aos períodos de ciclo no moinho.

Na Fig. 3 nota-se que a partir de $1 \mathrm{~h}$ no moinho o resíduo apresentou distribuição do tamanho de partículas aproximadamente igual. Sendo assim, neste trabalho adotouse o tratamento de $1 \mathrm{~h}$, correspondendo a 1.800 ciclos. Neste estágio, o resíduo tem aproximadamente $90 \%$ passante na peneira ABNT 200. As partículas dentro dessa faixa de distribuição do tamanho de partículas são normalmente denominadas filler na constituição das argamassas e concretos. O filler tem partículas de dimensões da ordem da do cimento portland [18]. Esses podem, em função do meio ao qual serão inseridos e da sua constituição mineralógica, atuar como materiais inertes ou não. O resíduo cerâmico atua como material pozolânico, reagindo durante o processo de hidratação do cimento, com o $\mathrm{Ca}^{2+}$ que está presente nesta hidratação, evitando a formação de portlandita $(\mathrm{CaOH})$, que

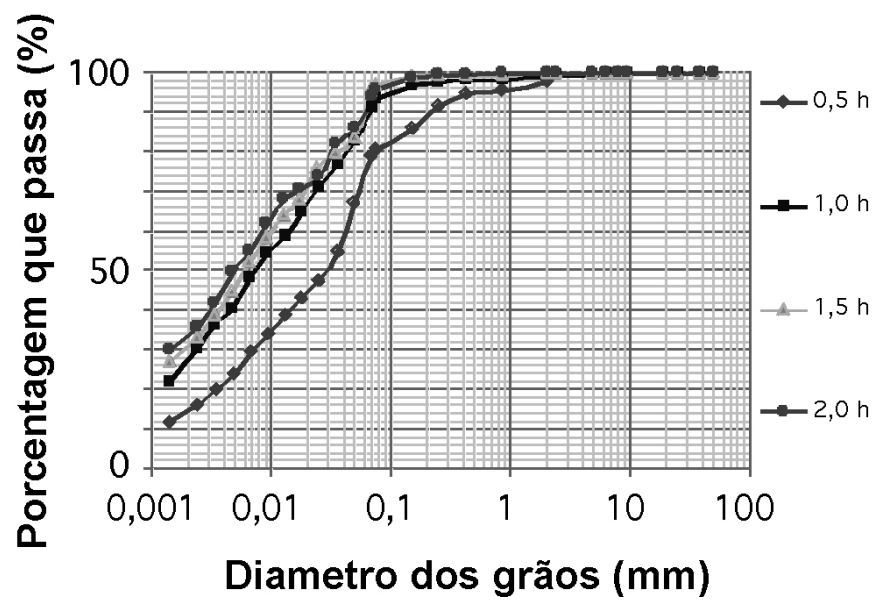

Figura 3: Curvas da distribuição do tamanho de partículas correspondentes aos tempos de ciclo no moinho.

[Figure 3: Particle size distribution curves related to the cycle time in the mill.] 
é a parte mais frágil da matriz cimentícia do argamassa. Logo, o resíduo é classificado como não inerte.

A seguir apresenta-se na Tabela $I$ e na Fig. 4 a composição química [10] e o difratograma de raios X de uma argila típica da região de Campos dos Goytacazes, [12] respectivamente.

Analisando a composição química do resíduo cerâmico, observa-se a quantidade sílica + alumina $>83 \%$, quantidade de $\mathrm{Fe}_{2} \mathrm{O}_{3}>8 \%$, quantidade de $\mathrm{TiO}_{2}>1,5 \%$ e $\mathrm{K}_{2} \mathrm{O}>1 \%$, que contribuem para a formação de novos cristais na matriz cimentícia da argamassa.

Os difratogramas de raios X, Fig. 4, mostram o inicio da fase amorfa da metacaulinita em torno de $490{ }^{\circ} \mathrm{C}$ com a ausênica dos picos cristalinos característicos da caulinita [12]. Ressalta-se ainda o fim da fase amorfa do material e inicio do processo de recristalização, característico de mulita acima de $900{ }^{\circ} \mathrm{C}$ e melhor observada a $1100^{\circ} \mathrm{C}$, acompanhada também da formação de cristobalita e de quatzo remanescente da matéria-prima. Os materiais cerâmicos vermelhos das indústrias são calcinados entre $600{ }^{\circ} \mathrm{C}$ e $800{ }^{\circ} \mathrm{C}$, onde a metacaulinita (fase amorfa) está presente, observada esta

Tabela I - Composição química do resíduo cerâmico.

[Table I - Chemical composition of the ceramic waste.]

\begin{tabular}{cc}
\hline Elemento & Quantidade (\%) \\
\hline $\mathrm{SiO}_{2}$ & 45,9 \\
$\mathrm{Al}_{2} \mathrm{O}_{3}$ & 38,0 \\
$\mathrm{Fe}_{2} \mathrm{O}_{3}$ & 8,5 \\
$\mathrm{SO}_{3}$ & 2,3 \\
$\mathrm{TiO}_{2}$ & 1,7 \\
$\mathrm{~K}_{2} \mathrm{O}$ & 2,5 \\
Outros & 1,1 \\
\hline
\end{tabular}

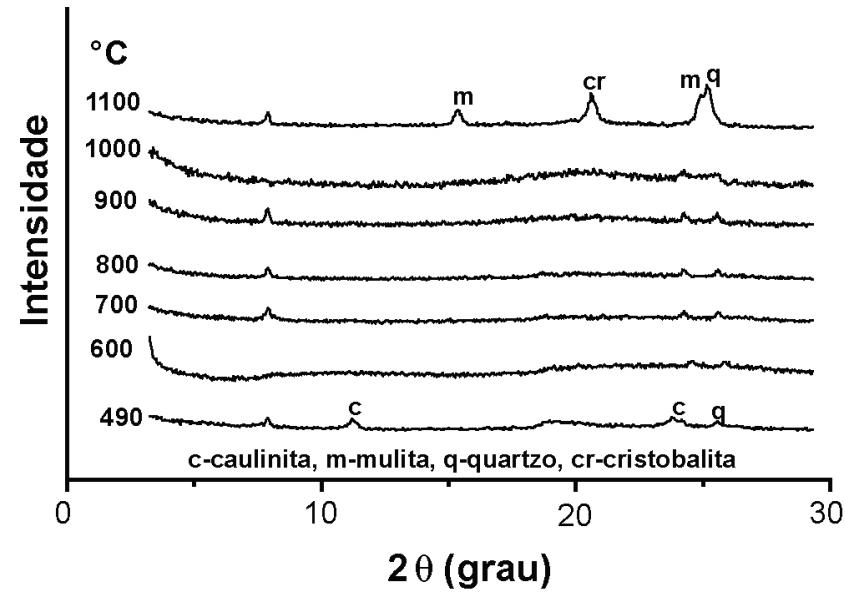

Figura 4: Difratograma de argila típica da região de Campos dos Goytacazes, RJ

[Figure 4: X-ray diffraction pattern of the typical clay of the Campos dos Goytacazes region.]

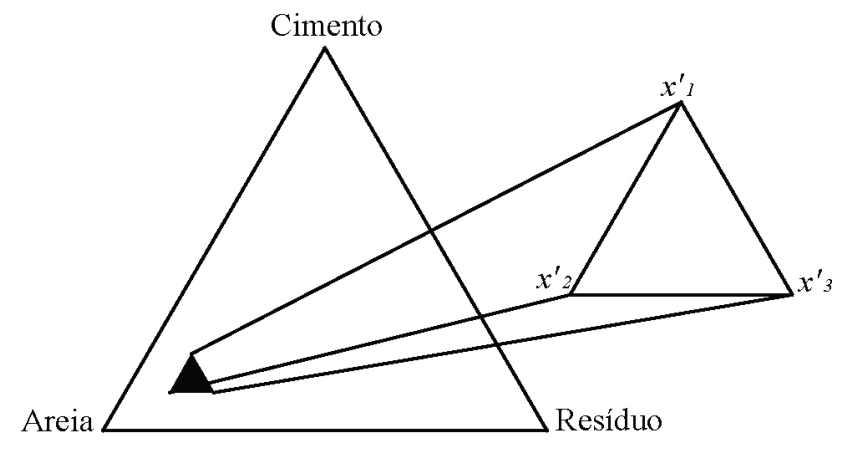

Figura 5: Região delimitada pelos pseudocomponentes. [Figure 5: Delimited area by the pseudocomponents.]

Tabela II - Nomenclatura usada em misturas para $\mathrm{q}=3$ componentes.

[Table II - Used Nomenclature in component mix.]

\begin{tabular}{|c|c|c|c|c|c|c|c|}
\hline \multirow[t]{2}{*}{$\begin{array}{c}\text { Pontos } \\
\text { experimentais }\end{array}$} & \multicolumn{3}{|c|}{$\begin{array}{c}\text { Matriz de } \\
\text { composição } \\
\text { das misturas - } X \\
\text { (pseudocomponetes) }\end{array}$} & Cimento & \multicolumn{2}{|c|}{ Componetes originais } & \multirow{2}{*}{$\begin{array}{c}\text { Respostas }(\bar{y}) \\
\text { Matriz das } \\
\text { respostas - y }\end{array}$} \\
\hline & $x_{1}$ & $x_{2}^{\prime}$ & $x_{3}^{\prime}$ & & & & \\
\hline$x_{1}$ & 1 & 0 & 0 & 0,20 & 0,70 & 0,10 & $y_{1}$ \\
\hline$x_{2}$ & 0 & 1 & 0 & 0,10 & 0,80 & 0,10 & $\boldsymbol{y}_{2}$ \\
\hline$x_{3}^{2}$ & 0 & 0 & 1 & 0,10 & 0,70 & 0,20 & $y_{3}$ \\
\hline$x_{12}$ & $1 / 2$ & $1 / 2$ & 0 & 0,15 & 0,75 & 0,10 & $\boldsymbol{y}_{12}$ \\
\hline$x_{13}$ & $1 / 2$ & 0 & $1 / 2$ & 0,15 & 0,70 & 0,15 & $\boldsymbol{y}_{13}$ \\
\hline$x_{23}$ & 0 & $1 / 2$ & $1 / 2$ & 0,10 & 0,75 & 0,15 & $\boldsymbol{y}_{23}$ \\
\hline$x_{123}^{20}$ & $1 / 3$ & $1 / 3$ & $1 / 3$ & 0,13 & 0,73 & 0,13 & $\boldsymbol{y}_{123}$ \\
\hline $\boldsymbol{x}_{1123}$ & $2 / 3$ & $1 / 6$ & $1 / 6$ & 0,17 & 0,72 & 0,12 & $\boldsymbol{y}_{1123}$ \\
\hline$x_{1223}$ & $1 / 6$ & $2 / 3$ & $1 / 6$ & 0,12 & 0,77 & 0,12 & $\boldsymbol{y}_{1223}$ \\
\hline $\boldsymbol{x}_{1233}$ & $1 / 6$ & $1 / 6$ & $2 / 3$ & 0,12 & 0,72 & 0,17 & $\boldsymbol{y}_{1233}$ \\
\hline
\end{tabular}


faixa de temperatura no difratograma. Pode-se então afirmar que o resíduo cerâmico possui atividade pozolânica devido à presença de fase amorfa.

A superfície de resposta, delimitada pelos pseudocomponentes para a consistência adotada, é ilustrada pela área em negrito da Fig. 5, que representa as restrições experimentais $\left(0,10 \leq \mathrm{X}_{1} \leq 0,20 ; 0,70 \leq \mathrm{x}_{2} \leq 0,80\right.$ e $\left.0,10 \leq \mathrm{X}_{3} \leq 0,20\right)$. Os pontos experimentais são distribuídos nesta área em negrito.

Os pseudocomponentes $\left(x_{1}^{\prime}, x_{2}^{\prime}{ }_{2} \mathrm{e} x_{3}^{\prime}\right)$ representam os pontos iniciais do planejamento experimental, onde as proporções em massa são $x^{\prime}{ }_{1}=20 \%$ de cimento $+70 \%$ de areia $+10 \%$ de resíduo, $x_{2}=10 \%$ de cimento $+80 \%$ de areia $+10 \%$ de resíduo e $x_{3}^{\prime}=10 \%$ de cimento $+70 \%$ de areia $+20 \%$ de resíduo.

Expandindo-se as matrizes representadas na equação $\mathrm{B}$ têm-se as equações dos modelos com seus respectivos parâmetros $b$ calculados. A Tabela II lista as nomenclaturas usadas para misturas de três componentes. Consideramse os pontos $\boldsymbol{x}_{1123}, \boldsymbol{x}_{1223}$ e $\boldsymbol{x}_{1233}$, que foram confeccionados para o teste dos modelos e, sendo assim, excedem os pontos necessários para a elaboração dos mesmos.

Seguem-se os modelos matemáticos gerados por meio do planejamento experimental, assim como um comparativo entre a eficiência dos modelos e análise estatística.
Modelo linear

$$
\hat{y}=92,37 x^{\prime}{ }_{1}+88,76 x_{2}^{\prime}+95,25 x_{3}^{\prime}
$$

\section{Modelo quadrático}

$\hat{y}=92,37 x^{\prime}{ }_{1}+88,76 x_{2}^{\prime}+95,25 x_{3}^{\prime}-6,00 x_{1}{ }_{1} x_{2}{ }_{2}$ $-2,02 x_{1}^{\prime} x_{3}^{\prime}+4,70 x_{2}{ }_{2} x_{3}{ }_{3}$

\section{Modelo cúbico especial}

$\hat{y}=92,37 x^{\prime}{ }_{1}+88,76 x_{2}{ }_{2}+95,25 x^{\prime}{ }_{3}-6,00 x^{\prime}{ }_{1} x_{2}{ }_{2}-$

$2,02 x_{1}^{\prime} x_{3}^{\prime}+4,70 x_{2}^{\prime} x_{3}^{\prime}+25,87 x_{1}^{\prime} x_{2}^{\prime} x_{3}^{\prime}$

Os componentes constituintes da argamassa, cimento, areia e resíduo cerâmico, representados nos vértices do triangulo da superfície de resposta da Fig. 6 correspondem às proporções máximas de 20,80 e $20 \%$, respectivamente em massa, conforme as restrições experimentais. As curvas de níveis ou de iso-respostas apresentam mesma capacidade de retenção de água, existindo várias possibilidades de proporção dos componentes. Usando-se uma maior quantidade de resíduo cerâmico para uma determinada curva

Tabela III - Valores previstos pelo modelo linear $(\hat{y})$ e valores médios de observações $(\bar{y})$.

[Table III - Planned values by the lineal model $(\hat{y})$ and medium observed values $(\bar{y})$.]

\begin{tabular}{ccccccccccc}
\hline Respostas & $x_{1}$ & $x_{2}$ & $x_{3}$ & $x_{12}$ & $x_{13}$ & $x_{23}$ & $x_{123}$ & $x_{1123}$ & $x_{1223}$ & $x_{1233}$ \\
\hline$\hat{y}$ & 92,37 & 88,76 & 95,25 & 90,56 & 93,81 & 92,01 & 92,13 & 92,24 & 90,45 & 93,67 \\
$\bar{y}$ & 92,37 & 88,77 & 95,26 & 89,07 & 93,31 & 93,19 & 92,72 & 91,77 & 92,24 & 92,24 \\
\hline
\end{tabular}

Tabela IV - ANOVA do modelo linear.

[Table IV - Linear model ANOVA.]

\begin{tabular}{ccccc}
\hline Fonte de variação & Soma quadrática & $\begin{array}{c}\mathrm{N}^{\mathrm{o}} \text { de graus de } \\
\text { liberdade }\end{array}$ & Média quadrática & $\begin{array}{c}\text { Teste F 95\% de } \\
\text { probabilidade }\end{array}$ \\
\hline Regressão & 94,43 & 2 & 47,21 & $M Q_{R} / M Q_{r}=1,54$ \\
Resíduo experimental & 61,21 & 27 & 2,67 & $F_{2,27}=3,35$ \\
Total & 155,64 & 29 & 5,37 & $M Q_{R} / M Q_{r}<F_{2,27}$ \\
\hline
\end{tabular}

Coeficiente de determinação: $R^{2}=S Q_{R} / S Q_{T}=60,67 \%$

Tabela $\mathrm{V}$ - Estimativa do modelo quadrático $(\hat{y})$ e valores médios de observações $(\bar{y})$.

[Table V-Estimated quadratic model $(\hat{y})$ and medium observed values $(\bar{y})$.]

\begin{tabular}{ccccccccccc}
\hline Respostas & $x_{1}$ & $x_{2}$ & $x_{3}$ & $x_{12}$ & $x_{13}$ & $x_{23}$ & $x_{123}$ & $x_{1123}$ & $x_{1223}$ & $x_{1233}$ \\
\hline$\hat{y}$ & 92,37 & 88,76 & 95,25 & 90,56 & 93,81 & 92,01 & 91,76 & 91,49 & 90,24 & 93,82 \\
$\bar{y}$ & 92,37 & 88,77 & 95,26 & 89,07 & 93,31 & 93,19 & 92,72 & 91,77 & 92,24 & 92,24 \\
\hline
\end{tabular}


Tabela VI - ANOVA do modelo quadrático.

[Table VI - Quadratic model ANOVA.]

\begin{tabular}{ccccc}
\hline Fonte de Variação & Soma quadrática & $\begin{array}{c}\mathrm{N}^{\mathrm{o}} \text { de graus de } \\
\text { liberdade }\end{array}$ & Média quadrática & $\begin{array}{c}\text { Teste F 95\% de } \\
\text { probabilidade }\end{array}$ \\
\hline Regressão & 104,27 & 5 & 20,85 & $M Q_{R} / M Q_{r}=2,03$ \\
Resíduo experimental & 51,36 & 24 & 2,14 & $F_{5,24}=2,10$ \\
Total & 155,63 & 29 & 5,37 & $M Q_{R} / M Q_{r}<. F_{2,27}$ \\
\hline
\end{tabular}

Coeficiente de determinação: $R^{2}=S Q_{R} / S Q_{T}=66,99 \%$

Tabela VII - Previsão do modelo cúbico especial ( $\hat{y})$ e valores médios de observações $(\bar{y})$.

[Table VII - Forecast of the special cubic model $(\hat{y})$ and medium values of observations $(\bar{y})$.]

\begin{tabular}{ccccccccccc}
\hline Respostas & $x_{1}$ & $x_{2}$ & $x_{3}$ & $x_{12}$ & $x_{13}$ & $x_{23}$ & $x_{123}$ & $x_{1123}$ & $x_{1223}$ & $x_{1233}$ \\
\hline$\hat{y}$ & 92,37 & 88,76 & 95,25 & 90,56 & 93,81 & 92,01 & 92,13 & 91,97 & 90,72 & 94,30 \\
$\bar{y}$ & 92,37 & 88,77 & 95,26 & 89,07 & 93,31 & 93,19 & 92,72 & 91,77 & 92,24 & 92,24 \\
\hline
\end{tabular}

Tabela VIII - ANOVA do modelo cúbico especial

[Table VIII - Special cubic model ANOVA.]

\begin{tabular}{|c|c|c|c|c|}
\hline Fonte de variação & Soma quadrática & $\begin{array}{l}\mathrm{N}^{\circ} \text { de graus de } \\
\text { liberdade }\end{array}$ & Média quadrática & $\begin{array}{l}\text { Teste F } 95 \% \text { de } \\
\text { probabilidade }\end{array}$ \\
\hline Regressão & 94,43 & 6 & 17,88 & $M Q_{R} / M Q_{r}=2,98$ \\
\hline Resíduo experimental & 61,21 & 3 & 2,10 & $F_{6,29}=2,43$ \\
\hline Total & 155,64 & 29 & 5,37 & $M Q_{R} / M Q_{r}>F_{2,27}$ \\
\hline
\end{tabular}

Coeficiente de determinação: $R^{2}=S Q_{R} / S Q_{T}=68,94 \%$

Tabela IX - Comparação entre os valores médios observados $(\bar{y})$ e previstos $(\hat{y})$

\begin{tabular}{|c|c|c|c|c|c|c|}
\hline \multicolumn{3}{|c|}{$\begin{array}{l}\text { Composição das Misturas } \\
\text { (Matriz das Misturas - X) }\end{array}$} & \multirow{2}{*}{$\begin{array}{c}\bar{y} \\
\text { (Observado) }\end{array}$} & \multirow{2}{*}{$\begin{array}{c}\hat{y} \\
\text { (Linear) }\end{array}$} & \multirow{2}{*}{$\begin{array}{c}\hat{y} \\
\text { (Quadrático) }\end{array}$} & \multirow{2}{*}{$\begin{array}{c}\hat{y} \\
\text { Cúbico } \\
\text { especial }\end{array}$} \\
\hline$x^{\prime}{ }_{1}$ & $x_{2}^{\prime}$ & $x_{3}$ & & & & \\
\hline 1 & 0 & 0 & 92,37 & 92,37 & 92,37 & 92,37 \\
\hline 0 & 1 & 0 & 88,77 & 88,77 & 88,77 & 88,77 \\
\hline 0 & 0 & 1 & 95,26 & 95,26 & 95,26 & 95,26 \\
\hline $1 / 2$ & $1 / 2$ & 0 & 89,07 & 90,56 & 89,07 & 89,07 \\
\hline $1 / 2$ & 0 & $1 / 2$ & 93,31 & 93,81 & 93,31 & 93,31 \\
\hline 0 & $1 / 2$ & $1 / 2$ & 93,19 & 92,01 & 93,19 & 93,19 \\
\hline $1 / 3$ & $1 / 3$ & $1 / 3$ & 92,72 & 92,13 & 91,76 & 92,72 \\
\hline $2 / 3$ & $1 / 6$ & $1 / 6$ & 91,77 & 92,24 & 91,49 & 91,97 \\
\hline $1 / 6$ & $2 / 3$ & $1 / 6$ & 92,24 & 90,45 & 90,24 & 90,72 \\
\hline $1 / 6$ & $1 / 6$ & $2 / 3$ & 94,20 & 93,67 & 93,82 & 94,30 \\
\hline \multicolumn{3}{|c|}{$\begin{array}{c}\text { Soma dos Resíduos } \\
\text { experimentais deixados } \\
\text { pelos modelos }\end{array}$} & $\sum e$ & 6,55 & 3,62 & 1,82 \\
\hline \multicolumn{3}{|c|}{$\begin{array}{l}\text { Média dos Resíduos } \\
\text { experimentais }\end{array}$} & $\bar{e}$ & 0,41 & 0,22 & 0,11 \\
\hline
\end{tabular}



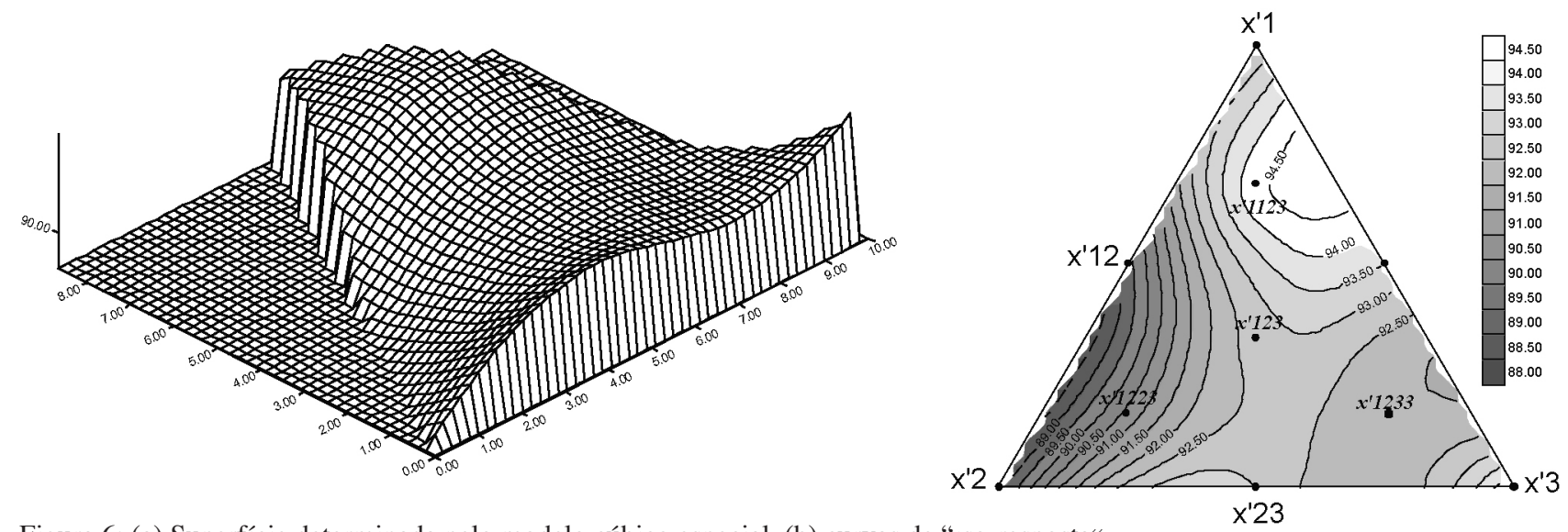

Figura 6: (a) Superfície determinada pelo modelo cúbico especial, (b) curvas de "1so-resposta".

[Figure 6: (a) Special cubic model surface, (b) “iso-response” curves.]

de nível, não se altera a capacidade de retenção de água. A superfície de resposta gerada apresentou capacidade de retenção de água da argamassa variando de $88 \%$ a $94,5 \%$, sendo classificada como normal e alta segundo a norma ABNT 13281. Conclui-se então, mesmo com valor superior ao índice de consistência padrão (valor acima estabelecido por norma) conseguiu-se uma capacidade de retenção padronizada com adição de resíduo na argamassa.

\section{CONCLUSÕES}

Para a consistência de $265 \pm 30 \mathrm{~mm}$ o modelo cúbico especial se ajusta melhor aos resultados experimentais permitindo estimar respostas estatisticamente mais precisas para a região delimitada por seus pseudocomponentes, deixando valores menores de resíduos experimentais. Analisando a superfície de resposta gerada, pode-se determinar de forma otimizada os traços pelos quais se podem obter as argamassas classificadas de normal ( $\geq 80 \%$ $\leq$ e $90 \%)$ ou alta (>90\%) capacidade de retenção de água [19].

Utilizando-se a superfície de resposta gerada no planejamento experimental, podem-se determinar faixas de misturas contínuas para um mesmo valor da propriedade, aumentando assim todas as possíveis faixas de proporção dos materiais para a consistência adotada, principalmente aumentando a adição de resíduo cerâmico, reduzindo o custo e mantendo o mesmo desempenho.

Vale ressaltar que as restrições experimentais deste trabalho são devidas ao consumo excessivo de água necessário para se obter a trabalhabilidade de uma argamassa de uso geral pelo fato do resíduo em questão absorver uma quantidade elevada de água.

\section{REFERÊNCIAS}

[1] F. Saboya Jr., J. Alexandre, R. Toledo, "Aplicação do planejamento em rede simplex para análise de matériaprima em cerâmicas vermelhas", $44^{\circ}$ Congresso Brasileiro de Cerâmica, S. Pedro 3 (2000) 1-6.
[2] J. A. Cornell, Experiments with mixtures: designs, models, and the analysis of mixtures data, $2^{\text {nd }} \mathrm{Ed}$. (1990).

[3] B. Barros Neto, I. S. Spacino, R. E. Bruns, Como fazer experimentos: pesquisa e desenvolvimento na indústria, $2^{\mathrm{a}}$ Ed., Editora da Unicamp, Campinas, SP (2003).

[4] E. V. C. Galdamez, L. C. R. Carpinetti, Aplicação das técnicas de planejamento e análise de experimentos no processo de injeção plástica, Gestão e Produção 11, 1 (2004) 121-134.

[5] J. Alexandre, "Análise da Matéria-Prima e Composição de Massa Utilizada em Cerâmica Vermelha", Tese de Doutorado, Universidade Estadual do Norte Fluminense Darcy Ribeiro, UENF, Campos dos Goytacazes, RJ (2000). [6] Associação Brasileira de Normas Técnicas - ABNT, “Agregados para concreto", Especificação - NBR 7211. Rio de Janeiro, RJ (1983).

[7] Associação Brasileira de Normas Técnicas - ABNT, "Agregado em estado solto - Determinação da massa unitária - Método de ensaio" - NBR 7251, Rio de Janeiro, RJ (1982).

[8] Associação Brasileira de Normas Técnicas - ABNT, "Areia Normal para Ensaio de Cimento", NBR 7214. Rio de Janeiro, RJ (1982).

[9] Associação Brasileira de Normas Técnicas - ABNT, “Amostragem de Resíduos: Procedimento", NBR 10007, Rio de Janeiro, RJ (1987).

[10] B. C. Costa, J. Alexandre, E. S. Freitas, J. M. Désir, D. P. Dias, Caracterização do resíduo cerâmico do município de Campos dos Goytacazes como material pozolânico em matrizes cimentíceas, $49^{\circ}$ Congresso Brasileiro de Cerâmica, S. Pedro, SP (2005) 1-12.

[11] G. C. Xavier, "Resistência, alterabilidade e durabilidade de peças cerâmicas vermelhas incorporadas com resíduo de granito", Tese de Doutorado, Universidade Estadual do Norte Fluminense Darcy Ribeiro, UENF, Campos dos Goytacazes, RJ (2006).

[12] L. P. Mothé, "Determinação do processo ideal de produção da metacaulinita por meio da análise de sua atividade pozolânica", Diss. Mestrado, Universidade Estadual do Norte Fluminense Darcy Ribeiro, UENF, 
Campos dos Goytacazes, RJ (2001).

[13] G. C. Isaia, P. R. L. Helene, Durabilidade de concreto de elevado desempenho com pozolanas, Anais Seminário Qualidade e Durabilidade das Estruturas de Concreto, Porto Alegre, RS (1993) 13-33.

[14] Associação Brasileira de Normas Técnicas - ABNT, "Argamassas para assentamento de paredes e revestimento de paredes e tetos - Determinação da retenção de água", NBR 13277, Rio de Janeiro, RJ (1995).

[15] Associação Brasileira de Normas Técnicas - ABNT, "Argamassas para assentamento de paredes e revestimento de paredes e tetos - Determinação do teor de água para a obtenção do índice de consistência padrão", NBR 13276 (1995).

[16] J. M. Désir, D. P. Dias, C. A. A. Rocha, D. F. Dantas,
Avaliação da Contribuição da Atividade Pozolânica do Resíduo de Tijolo Moído na Resistência de Argamassas, Conf. Interam. Materiais e Tecnologias não-convencionais na Construção Ecológica e Sustentável, Rio de Janeiro, RJ (2005).

[17] Associação Brasileira de Normas Técnicas - ABNT, "Cimento Portland Composto", NBR 11578, Rio de Janeiro, RJ (1991).

[18] E. G. R. Petruci, Concreto de Cimento Portland, 13 ${ }^{\mathrm{a}}$ Ed., Ed. Globo, S. Paulo, SP (1998).

[19] Associação Brasileira de Normas Técnicas - ABNT, "Argamassas para assentamento de paredes e revestimento de paredes e tetos - Requisitos", NBR 13281, Rio de Janeiro, RJ (2001).

(Rec. 02/08/2007, Rev. 16/04/2008, Ac. 25/04/2008) 